\title{
DILOGARITHM IDENTITIES FOR CONFORMAL FIELD THEORIES AND CLUSTER ALGEBRAS: SIMPLY LACED CASE
}

\author{
TOMOKI NAKANISHI
}

\begin{abstract}
The dilogarithm identities for the central charges of conformal field theories of simply laced type were conjectured by Bazhanov, Kirillov, and Reshetikhin. Their functional generalizations were conjectured by Gliozzi and Tateo. They have been partly proved by various authors. We prove these identities in full generality for any pair of Dynkin diagrams of simply laced type based on the cluster algebra formulation of the Y-systems.
\end{abstract}

\section{$\S 1$. Introduction}

\subsection{Dilogarithm identities}

Let $L(x)$ be the Rogers dilogarithm function (see [L], [K2], [Zag2], [N]):

$$
L(x)=-\frac{1}{2} \int_{0}^{x}\left\{\frac{\log (1-y)}{y}+\frac{\log y}{1-y}\right\} d y \quad(0 \leq x \leq 1) .
$$

It is well known that the following properties hold $(0 \leq x, y \leq 1)$ :

$$
\begin{gathered}
L(0)=0, \quad L(1)=\frac{\pi^{2}}{6}, \\
L(x)+L(1-x)=\frac{\pi^{2}}{6}, \\
L(x)+L(y)+L(1-x y)+L\left(\frac{1-x}{1-x y}\right)+L\left(\frac{1-y}{1-x y}\right)=\frac{\pi^{2}}{2} .
\end{gathered}
$$

In the series of works by Bazhanov, Kirillov, and Reshetikhin ([KR1], [BR1], [KR2], [K1], [BR2]), the authors reached a remarkable conjecture on identities expressing the central charges of conformal field theories in terms of $L(x)$ and partly established it. Let us concentrate on the identities in the simply laced case here.

Received October 7, 2009. Revised May 10, 2010. Accepted July 19, 2010.

2010 Mathematics Subject Classification. Primary 13F60; Secondary 17 B37.

(C) 2011 by The Editorial Board of the Nagoya Mathematical Journal 
Let $X_{r}$ be any simply laced Dynkin diagram of finite type with rank $r$, and let $I$ be the index set of $X_{r}$. Let $\ell \geq 2$ be any integer. For a family of positive real numbers $\left\{Y_{m}^{(a)} \mid a \in I ; 1 \leq m \leq \ell-1\right\}$, consider a system of algebraic relations

$$
\left(Y_{m}^{(a)}\right)^{2}=\frac{\prod_{b: b \sim a}\left(1+Y_{m}^{(b)}\right)}{\left(1+Y_{m-1}^{(a)}-1\right)\left(1+Y_{m+1}^{(a)}-1\right)},
$$

where $b \sim a$ means that $b$ is adjacent to $a$ in $X_{r}$, and $Y_{0}^{(a)}-1=Y_{\ell}^{(a)}-1=0$ if they appear in the right-hand sides.

TheOREM 1.1 (see [NK], [Zag2]). There exists a unique positive real solution of (1.5).

Conjecture 1.2 (dilogarithm identities (see [K1], [BR2])). Suppose that a family of positive real numbers $\left\{Y_{m}^{(a)} \mid a \in I ; 1 \leq m \leq \ell-1\right\}$ satisfies (1.5). Then, we have the identities

$$
\frac{6}{\pi^{2}} \sum_{a \in I} \sum_{m=1}^{\ell-1} L\left(\frac{Y_{m}^{(a)}}{1+Y_{m}^{(a)}}\right)=\frac{\ell \operatorname{dim} \mathfrak{g}}{h+\ell}-r,
$$

where $h$ and $\mathfrak{g}$ are the Coxeter number and the simple Lie algebra of type $X_{r}$, respectively.

REMARK 1.3. The conjectures in [K1] and [BR2] are not exactly the same. This is the version in $[\mathrm{BR} 2,(4.21),(4.22),(5.3)]$ with the identification of $f_{j}^{a}$ therein with $Y_{j}^{(a)} /\left(1+Y_{j}^{(a)}\right)$ here. The version in [K1] also concerns the construction of the solution of (1.5). We do not touch this issue here, since it is regarded as an independent problem in the framework of the present paper.

For $X_{r}=A_{r}$, Kirillov [K1] gave the explicit expression of the solution of (1.5) and proved the corresponding identity (1.6) by the analytic method.

Due to the well-known formula $\operatorname{dim} \mathfrak{g}=r(h+1)$, the right-hand side of (1.6) is equal to the number

$$
\frac{(\ell-1) r h}{h+\ell}
$$

It is already remarkable that the left-hand side of (1.6) is a rational number. It is much more remarkable that the rational number of the first term in 
the right-hand side of (1.6) is the central charge of the Wess-Zumino-Witten conformal field theory (see [KnZ], [GW]) of type $X_{r}$ with level $\ell$. (This is one of the reasons that the integer $\ell$ is called the level.) The rational number in the right-hand side of (1.6) itself is also the central charge of the parafermion conformal field theory of type $X_{r}$ with level $\ell$ (see $[\mathrm{FZ}],[\mathrm{G}]$ ). The identity (1.6) is crucial to establish the connection between conformal field theories and various types of nonconformal integrable models in various limits.

ExAmple $1.4([\mathrm{KR} 1])$. Consider the case $X_{r}=A_{1}$ and any $\ell$, which is equivalent to the case $X_{r}=A_{\ell-1}$ and $\ell=2$ by the level-rank duality. Then, one has a solution of (1.5),

$$
Y_{m}^{(1)}=\frac{\sin ^{2}(\pi /(\ell+2))}{\sin (m \pi /(\ell+2)) \sin ((m+2) \pi /(\ell+2))},
$$

and the corresponding identity (1.6) reads

$$
\frac{6}{\pi^{2}} \sum_{m=1}^{\ell-1} L\left(\frac{\sin ^{2}(\pi /(\ell+2))}{\sin ^{2}((m+1) \pi /(\ell+2))}\right)=\frac{3 \ell}{2+\ell}-1 .
$$

This identity has been known and studied by various authors from various points of view (see, e.g., [L], [RS], [KR1], [NRT], [DS]).

\subsection{Functional dilogarithm identities}

The system (1.5) admits an affinization called the $Y$-system introduced by Zamolodchikov [Zam], Kuniba and Nakanishi [KuN], and Ravanini, Tateo, and Valleriani [RTV]. Here, we consider the version in [RTV]. Let $X_{r}$ and $X_{r^{\prime}}^{\prime}$ be a pair of simply laced Dynkin diagrams of finite type. Let $I$ and $I^{\prime}$ be the index sets of $X_{r}$ and $X_{r^{\prime}}^{\prime}$, respectively. For a family of variables $\left\{Y_{i i^{\prime}}(u) \mid i \in I, i^{\prime} \in I^{\prime}, u \in \mathbb{Z}\right\}$, the $Y$-system $\mathbb{Y}\left(X_{r}, X_{r^{\prime}}^{\prime}\right)$ associated with a pair $\left(X_{r}, X_{r^{\prime}}^{\prime}\right)$ is a system of the algebraic/functional relations

$$
Y_{i i^{\prime}}(u-1) Y_{i i^{\prime}}(u+1)=\frac{\prod_{j: j \sim i}\left(1+Y_{j i^{\prime}}(u)\right)}{\prod_{j^{\prime}: j^{\prime} \sim i^{\prime}}\left(1+Y_{i j^{\prime}}(u)^{-1}\right)},
$$

where $j \sim i$ means that $j$ is adjacent to $i$ in $X_{r}$, while $j^{\prime} \sim i^{\prime}$ means that $j^{\prime}$ is adjacent to $i^{\prime}$ in $X_{r^{\prime}}^{\prime}$. Two systems $\mathbb{Y}\left(X_{r}, X_{r^{\prime}}^{\prime}\right)$ and $\mathbb{Y}\left(X_{r^{\prime}}^{\prime}, X_{r}\right)$ are equivalent to each other by the correspondence $Y_{i i^{\prime}}(u) \leftrightarrow Y_{i^{\prime} i}(u)^{-1}$. This is a generalization of the level-rank duality.

Ravanini, Tateo, and Valleriani [RTV] gave the periodicity conjecture, which generalized the one by [Zam] in the case $X_{r}=A_{1}$ or $X_{r^{\prime}}^{\prime}=A_{1}$. 
Conjecture 1.5 (periodicity [RTV, Section 6]). Suppose that a family of positive real numbers $\left\{Y_{i i^{\prime}}(u) \mid i \in I, i^{\prime} \in I^{\prime}, u \in \mathbb{Z}\right\}$ satisfies $\mathbb{Y}\left(X_{r}, X_{r^{\prime}}^{\prime}\right)$. Then, we have the periodicity

$$
Y_{i i^{\prime}}\left(u+2\left(h+h^{\prime}\right)\right)=Y_{i i^{\prime}}(u),
$$

where $h$ and $h^{\prime}$ are the Coxeter numbers of types $X_{r}$ and $X_{r^{\prime}}^{\prime}$, respectively.

Conjecture 1.5 was proved for $\left(X_{r}, X_{r^{\prime}}^{\prime}\right)=\left(A_{r}, A_{1}\right)$ by Gliozzi and Tateo [GT2] and Frenkel and Szenes [FS], for $\left(X_{r}, X_{r^{\prime}}^{\prime}\right)=\left(\right.$ any,$\left.A_{1}\right)$ by Fomin and Zelevinsky [FZ3], and for $\left(X_{r}, X_{r^{\prime}}^{\prime}\right)=\left(A_{r}, A_{r^{\prime}}\right)$ by Volkov [V] and Szenes [S]. More recently, it was proved in full generality by Keller ([Ke1], [Ke2]).

Furthermore, Gliozzi and Tateo [GT1] significantly generalized Conjecture 1.2 as follows.

Conjecture 1.6 (functional dilogarithm identities (see [GT1])). Suppose that a family of positive real numbers $\left\{Y_{a a^{\prime}}(u) \mid a \in I, a^{\prime} \in I^{\prime}, u \in \mathbb{Z}\right\}$ satisfies $\mathbb{Y}\left(X_{r}, X_{r^{\prime}}^{\prime}\right)$. Then, we have the identities

$$
\begin{aligned}
& \frac{6}{\pi^{2}} \sum_{\left(i, i^{\prime}\right) \in I \times I^{\prime}} \sum_{u=0}^{2\left(h+h^{\prime}\right)-1} L\left(\frac{Y_{i i^{\prime}}(u)}{1+Y_{i i^{\prime}}(u)}\right)=2 h r r^{\prime}, \\
& \frac{6}{\pi^{2}} \sum_{\left(i, i^{\prime}\right) \in I \times I^{\prime}} \sum_{u=0}^{2\left(h+h^{\prime}\right)-1} L\left(\frac{1}{1+Y_{i i^{\prime}}(u)}\right)=2 h^{\prime} r r^{\prime} .
\end{aligned}
$$

The identities (1.12) and (1.13) are equivalent due to (1.3).

Conjecture 1.6 implies Conjecture 1.2; namely, set $X_{r^{\prime}}^{\prime}=A_{\ell-1}$, and take a constant solution $Y_{i i^{\prime}}=Y_{i i^{\prime}}(u)$ of $\mathbb{Y}\left(X_{r}, A_{\ell-1}\right)$ as a function of $u$. Then, one obtains (1.6) from (1.12) using $h^{\prime}=\ell, r^{\prime}=\ell-1$, and (1.7). Conjecture 1.6 was proved for $\left(X_{r}, X_{r^{\prime}}^{\prime}\right)=\left(A_{r}, A_{1}\right)$ by Frenkel and Szenes [FS], and for $\left(X_{r}, X_{r^{\prime}}^{\prime}\right)=\left(\right.$ any,$\left.A_{1}\right)$ by Chapoton $[\mathrm{C}]$.

EXAMPLE 1.7 (see [GT1]). (i) In the simplest case $\left(X_{r}, X_{r^{\prime}}^{\prime}\right)=\left(A_{1}, A_{1}\right)$, the identity (1.12) is equivalent to (1.3).

(ii) In the next simplest case $\left(X_{r}, X_{r^{\prime}}^{\prime}\right)=\left(A_{2}, A_{1}\right)$, the identity (1.12) is equivalent to the 5 -term relation (1.4).

Conjecture 1.6 tells us that the matters that are important in (1.6) are not the values of $Y_{m}^{(a)}$ themselves but rather the relations (1.10) that they satisfy. We will see below that the algebraic property of the relations (1.10) is efficiently extracted by cluster algebras introduced by Fomin and Zelevinsky ([FZ1], [FZ2], [FZ4]). 


\subsection{Main result}

Among several preceding results and methods concerning Conjectures 1.5 and 1.6, we list the ones which are particularly relevant to the present work.

(i) Frenkel and Szenes $[\mathrm{FS}]$ proved Conjecture 1.6 for $\left(X_{r}, X_{r^{\prime}}^{\prime}\right)=\left(A_{r}, A_{1}\right)$ by showing the constancy property of the left-hand side of (1.12).

(ii) Caracciolo, Gliozzi, and Tateo [CGT] studied the constancy property for any pair $\left(X_{r}, X_{r^{\prime}}^{\prime}\right)$. (But they did not complete the proof of Conjecture 1.6.)

(iii) Fomin and Zelevinsky [FZ3] proved Conjecture 1.5 for $\left(X_{r}, X_{r^{\prime}}^{\prime}\right)=$ (any, $A_{1}$ ) by using the "cluster algebra-like" formulation of Y-systems and the root systems.

(iv) Chapoton $[\mathrm{C}]$ proved Conjecture 1.6 for $\left(X_{r}, X_{r^{\prime}}^{\prime}\right)=\left(\right.$ any, $\left.A_{1}\right)$ by combining the constancy property of (i) and the result of (iii).

(v) Fomin and Zelevinsky [FZ4] more manifestly integrated the Y-system $\mathbb{Y}\left(X_{r}, A_{1}\right)$ in the framework of cluster algebras with coefficients, where the Y-system is identified with a system of relations among coefficients of the cluster algebras of type $X_{r}$.

(vi) Keller ([Ke1], [Ke2]) proved Conjecture 1.5 in full generality for any pair $\left(X_{r}, X_{r^{\prime}}^{\prime}\right)$ of simply laced type by using cluster algebras with coefficients of $(\mathrm{v})$ together with their categorifications by the cluster categories.

We also note that the connection between the dilogarithm and cluster algebras was studied earlier by Fock and Goncharov [FG].

By combining these results, methods, and ideas, we prove Conjecture 1.6.

TheOREM 1.8. Conjecture 1.6 is true for any pair $\left(X_{r}, X_{r^{\prime}}^{\prime}\right)$ of simply laced type.

Corollary 1.9. Conjecture 1.2 is true for any $X_{r}$ of simply laced type and any $\ell \geq 2$.

REMARK 1.10. The dilogarithm identities of nonsimply laced type by Kirillov $[\mathrm{K} 1,(7)]$, properly corrected by Kuniba $[\mathrm{Ku},(\mathrm{A} .1 \mathrm{a}),(\mathrm{A} .1 \mathrm{c})]$, are equally important. We stress that they are different from another version of the identities of nonsimply laced type obtained by the folding of simply laced one (see $[\mathrm{C}]$ ). Though the situation is more complicated than the simply laced case, a similar approach to the one here is applicable to prove the identities (see [IIKKN1], [IIKKN2]). 
The organization of the paper is the following. In Section 2, we reformulate Conjecture 1.6 in terms of cluster algebras. In Section 3, we study the tropical version of the Y-system in the cluster algebra setting. Proposition 3.2 is a key observation throughout the paper. In Section 4, we prove Theorem 2.8, which is equivalent to Theorem 1.8, by applying the method of $[\mathrm{FS}]$ with a mixture of the ideas by $[\mathrm{CGT}]$ and $[\mathrm{C}]$.

\section{$\S 2$. Reformulation by cluster algebras}

As the first step, we reformulate Theorem 1.8 in terms of cluster algebras.

\subsection{Cluster algebras}

Here we collect some basic definitions for cluster algebras (see [FZ1], [FZ2], [FZ4]) to fix the convention and notation, mainly following [FZ4].

(i) Matrix mutation. An integer matrix $B=\left(B_{i j}\right)_{i, j \in I}$ is skewsymmetrizable if there is a diagonal matrix $D=\operatorname{diag}\left(d_{i}\right)_{i \in I}$ with $d_{i} \in \mathbb{N}$ such that $D B$ is skew-symmetric. For a skew-symmetrizable matrix $B$ and $k \in I$, another matrix $B^{\prime}=\mu_{k}(B)$, called the mutation of $B$ at $k$, is defined by

$$
B_{i j}^{\prime}= \begin{cases}-B_{i j} & i=k \text { or } j=k \\ B_{i j}+\frac{1}{2}\left(\left|B_{i k}\right| B_{k j}+B_{i k}\left|B_{k j}\right|\right) & \text { otherwise. }\end{cases}
$$

The matrix $\mu_{k}(B)$ is also skew-symmetrizable.

(ii) Exchange relation of coefficient tuple. A semifield $(\mathbb{P}, \oplus)$ is an abelian multiplicative group $\mathbb{P}$ endowed with a binary operation of addition $\oplus$ which is commutative, associative, and distributive with respect to the multiplication in $\mathbb{P}$ (see [FZ4], [HW]). For an $I$-tuple $y=\left(y_{i}\right)_{i \in I}, y_{i} \in \mathbb{P}$ and $k \in I$, another $I$-tuple $y^{\prime}$ is defined by the exchange relation

$$
y_{i}^{\prime}= \begin{cases}y_{k}{ }^{-1} & i=k, \\ y_{i}\left(\frac{y_{k}}{1 \oplus y_{k}}\right)^{B_{k i}} & i \neq k, B_{k i} \geq 0 \\ y_{i}\left(1 \oplus y_{k}\right)^{-B_{k i}} & i \neq k, B_{k i} \leq 0\end{cases}
$$

(iii) Exchange relation of cluster. Let $\mathbb{Q P}$ be the quotient field of the group ring $\mathbb{Z P}$ of $\mathbb{P}$, and let $\mathbb{Q P}(u)$ be the rational function field of algebraically independent variables $u=\left(u_{i}\right)_{i \in I}$ over $\mathbb{Q P}$. 
For an $I$-tuple $x=\left(x_{i}\right)_{i \in I}$ which is a free generating set of $\mathbb{Q P}(u)$ and $k \in I$, another $I$-tuple $x^{\prime}$ is defined by the exchange relation

$$
x_{i}^{\prime}= \begin{cases}x_{k} & i \neq k, \\ \frac{y_{k} \prod_{j: B_{j k}>0} x_{j}^{B_{j k}}+\prod_{j: B_{j k}<0} x_{j}^{-B_{j k}}}{\left(1 \oplus y_{k}\right) x_{k}} & i=k .\end{cases}
$$

(iv) Seed mutation. For the above triplet $(B, x, y)$, called a seed, the mutation $\mu_{k}(B, x, y)=\left(B^{\prime}, x^{\prime}, y^{\prime}\right)$ at $k$ is defined by combining (i)-(iii).

(v) Cluster algebra. Fix a semifield $\mathbb{P}$ and a seed (initial seed) $(B, x, y)$, where $x=\left(x_{i}\right)_{i \in I}$ are algebraically independent variables over $\mathbb{Q P}$. Starting from $(B, x, y)$, iterate mutations and collect all the seeds $\left(B^{\prime}, x^{\prime}, y^{\prime}\right)$. We call $y^{\prime}$ and $y_{i}^{\prime}$ a coefficient tuple and a coefficient, respectively. We call $x^{\prime}$ and $x_{i}^{\prime} \in \mathbb{Q P}(x)$ a cluster and a cluster variable, respectively. The cluster algebra $\mathcal{A}(B, x, y)$ with coefficients in $\mathbb{P}$ is a $\mathbb{Z} \mathbb{P}$-subalgebra of the rational function field $\mathbb{Q P}(x)$ generated by all the cluster variables.

For further necessary definitions and information for cluster algebras, see [FZ4].

\subsection{Matrix $B\left(X_{r}, X_{r^{\prime}}^{\prime}\right)$}

For a Cartan matrix $C=\left(C_{i j}\right)_{i, j \in I}$ of finite type, we say that the decomposition $I=I_{+} \sqcup I_{-}$is bipartite if

$$
C_{i j}<0 \text {, then }(i, j) \in I_{+} \times I_{-} \text {or }(i, j) \in I_{-} \times I_{+} .
$$

From now on, we assume that $X_{r}$ and $X_{r^{\prime}}^{\prime}$ are a pair of simply laced Dynkin diagrams of finite type and that $C=\left(C_{i j}\right)_{i, j \in I}$ and $C^{\prime}=\left(C_{i^{\prime} j^{\prime}}\right)_{i^{\prime}, j^{\prime} \in I}$ are the Cartan matrices of $X_{r}$ and $X_{r^{\prime}}^{\prime}$ with fixed bipartite decompositions $I=I_{+} \sqcup I_{-}$and $I^{\prime}=I_{+}^{\prime} \sqcup I_{-}^{\prime}$, respectively. Set $\mathbf{I}=I \times I^{\prime}$. For $\mathbf{i}=\left(i, i^{\prime}\right) \in \mathbf{I}$, let us write $\mathbf{i}:(++)$ if $\left(i, i^{\prime}\right) \in I_{+} \times I_{+}^{\prime}$, and so forth. Define the matrix $B=B\left(X_{r}, X_{r^{\prime}}^{\prime}\right)=\left(B_{\mathbf{i j}}\right)_{\mathbf{i}, \mathbf{j} \in \mathbf{I}}$ by

$$
B_{\mathbf{i j}}= \begin{cases}-C_{i j} \delta_{i^{\prime} j^{\prime}} & \mathbf{i}:(-+), \mathbf{j}:(++) \text { or } \mathbf{i}:(+-), \mathbf{j}:(--), \\ C_{i j} \delta_{i^{\prime} j^{\prime}} & \mathbf{i}:(++), \mathbf{j}:(++) \text { or } \mathbf{i}:(--), \mathbf{j}:(+-), \\ -\delta_{i j} C_{i^{\prime} j^{\prime}}^{\prime} & \mathbf{i}:(++), \mathbf{j}:(+-) \text { or } \mathbf{i}:(--), \mathbf{j}:(-+), \\ \delta_{i j} C_{i^{\prime} j^{\prime}}^{\prime} & \mathbf{i}:(+-), \mathbf{j}:(++) \text { or } \mathbf{i}:(-+), \mathbf{j}:(--), \\ 0 & \text { otherwise }\end{cases}
$$


The rule (2.5) is visualized in the following diagram:

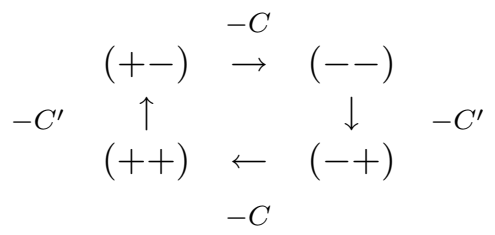

The matrix $B$ corresponds to the square product of alternating quivers by [Ke1].

Lemma 2.1. The matrix $B=B\left(X_{r}, X_{r^{\prime}}^{\prime}\right)$ in (2.5) is skew-symmetric and satisfies the following conditions. Let $\mathbf{I}=\mathbf{I}_{+} \sqcup \mathbf{I}_{-}$with $\mathbf{I}_{+}:=\left(I_{+} \times I_{+}^{\prime}\right) \sqcup$ $\left(I_{-} \times I_{-}^{\prime}\right)$ and $\mathbf{I}_{-}:=\left(I_{+} \times I_{-}^{\prime}\right) \sqcup\left(I_{-} \times I_{+}^{\prime}\right)$. Then,

$$
\text { if } B_{\mathbf{i j}} \neq 0 \text {, then }(\mathbf{i}, \mathbf{j}) \in \mathbf{I}_{+} \times \mathbf{I}_{-} \text {or }(\mathbf{i}, \mathbf{j}) \in \mathbf{I}_{-} \times \mathbf{I}_{+} \text {. }
$$

Furthermore, for composed mutations $\mu_{+}=\prod_{\mathbf{i} \in \mathbf{I}_{+}} \mu_{\mathbf{i}}$ and $\mu_{-}=\prod_{\mathbf{i} \in \mathbf{I}_{-}} \mu_{\mathbf{i}}$,

$$
\mu_{+}(B)=\mu_{-}(B)=-B
$$

Proof. They are easily seen in the quiver picture in [Ke1, Section 8].

Note that $\mu_{ \pm}(B)$ does not depend on the order of the product due to (2.7).

\subsection{Cluster algebra and $\mathrm{Y}$-system}

For the matrix $B=B\left(X_{r}, X_{r^{\prime}}^{\prime}\right)$ in $(2.5)$, let $\mathcal{A}(B, x, y)$ be the cluster algebra with coefficients in the universal semifield $\mathbb{Q}_{\mathrm{sf}}(y)$, where $(B, x, y)$ is the initial seed (see [FZ4]). (Here we use the symbol + instead of $\oplus$ in $\mathbb{Q}_{\text {sf }}(y)$, since it is the ordinary addition of subtraction-free expressions of rational functions of $y$.)

To our purpose, it is natural to introduce not only the "ring of cluster variables" but also the "group of coefficients."

Definition 2.2. The coefficient group $\mathcal{G}(B, y)$ associated with $\mathcal{A}(B, x, y)$ is the multiplicative subgroup of the semifield $\mathbb{Q}_{\mathrm{sf}}(y)$ generated by all the coefficients $y_{\mathbf{i}}^{\prime}$ of $\mathcal{A}(B, x, y)$ together with $1+y_{\mathbf{i}}^{\prime}$.

We set $x(0)=x$ and $y(0)=y$, and we define clusters $x(u)=\left(x_{\mathbf{i}}(u)\right)_{\mathbf{i} \in \mathbf{I}}$ $(u \in \mathbb{Z})$ and coefficient tuples $y(u)=\left(y_{\mathbf{i}}(u)\right)_{\mathbf{i} \in \mathbf{I}}(u \in \mathbb{Z})$ by the sequence of mutations

$$
\begin{aligned}
\cdots & \stackrel{\mu_{-}}{\longleftrightarrow}(B, x(0), y(0)) \stackrel{\mu_{+}}{\longleftrightarrow}(-B, x(1), y(1)) \\
& \stackrel{\mu_{-}}{\longleftrightarrow}(B, x(2), y(2)) \stackrel{\mu_{+}}{\longleftrightarrow} \cdots .
\end{aligned}
$$


Definition 2.3. The $Y$-subgroup $\mathcal{G}_{Y}(B, y)$ of $\mathcal{G}(B, y)$ associated with the sequence (2.9) is the multiplicative subgroup of $\mathcal{G}(B, y)$ generated by $y_{\mathbf{i}}(u)$ and $1+y_{\mathbf{i}}(u)(\mathbf{i} \in \mathbf{I}, u \in \mathbb{Z})$.

Let $\varepsilon: \mathbf{I} \rightarrow\{+,-\}$ be the sign function defined by $\varepsilon(\mathbf{i})=\varepsilon$ for $\mathbf{i} \in \mathbf{I}_{\varepsilon}$. For $(\mathbf{i}, u) \in \mathbf{I} \times \mathbb{Z}$, we set the "parity conditions" $\mathbf{P}_{+}$and $\mathbf{P}_{-}$by

$$
\mathbf{P}_{ \pm}: \quad \varepsilon(\mathbf{i})(-1)^{u}= \pm,
$$

where we identify + and - with 1 and -1 , respectively. We write $(\mathbf{i}, u): \mathbf{P}_{\varepsilon}$ if $(\mathbf{i}, u)$ satisfies the condition $\mathbf{P}_{\varepsilon}$.

Lemma 2.4 ([KuNS, Lemma 6.18]). (1) $y_{\mathbf{i}}(u)=y_{\mathbf{i}}(u \pm 1)^{-1}$ for $(\mathbf{i}, u): \mathbf{P}_{ \pm}$.

(2) The family $y_{ \pm}=\left\{y_{\mathbf{i}}(u) \mid(\mathbf{i}, u): \mathbf{P}_{ \pm}\right\}$satisfies the $Y$-system $\mathbb{Y}\left(X_{r}, X_{r^{\prime}}^{\prime}\right)$ in $\mathcal{G}_{Y}(B, y)$ by replacing $Y_{\mathbf{i}}(u)$ in $\mathbb{Y}\left(X_{r}, X_{r^{\prime}}^{\prime}\right)$ with $y_{\mathbf{i}}(u)^{ \pm 1}$.

Proof. This follows from the exchange relation (2.2).

Definition 2.5. Let $y\left(X_{r}, X_{r^{\prime}}^{\prime}\right)$ be the semifield with generators $Y_{\mathbf{i}}(u)$ $(\mathbf{i} \in \mathbf{I}, u \in \mathbb{Z})$ and the relations $\mathbb{Y}\left(X_{r}, X_{r^{\prime}}^{\prime}\right)$. Let $y^{\circ}\left(X_{r}, X_{r^{\prime}}^{\prime}\right)$ be the multiplicative subgroup of $y\left(X_{r}, X_{r^{\prime}}^{\prime}\right)$ generated by $Y_{\mathbf{i}}(u), 1+Y_{\mathbf{i}}(u)(\mathbf{i} \in \mathbf{I}, u \in \mathbb{Z})$. (Here we use the symbol + instead of $\oplus$ for simplicity.)

Define $y^{\circ}\left(X_{r}, X_{r^{\prime}}^{\prime}\right)_{\varepsilon}(\varepsilon= \pm)$ to be the subgroup of $y^{\circ}\left(X_{r}, X_{r^{\prime}}^{\prime}\right)$ generated by those $Y_{\mathbf{i}}(u), 1+Y_{\mathbf{i}}(u)$ with $(\mathbf{i}, u): \mathbf{P}_{\varepsilon}$. Then, we have $\mathrm{y}^{\circ}\left(X_{r}, X_{r^{\prime}}^{\prime}\right)_{+} \simeq$ $\mathrm{y}^{\circ}\left(X_{r}, X_{r^{\prime}}^{\prime}\right)_{-}$by $Y_{\mathbf{i}}(u) \mapsto Y_{\mathbf{i}}(u+1)$ and

$$
y^{\circ}\left(X_{r}, X_{r^{\prime}}^{\prime}\right) \simeq y^{\circ}\left(X_{r}, X_{r^{\prime}}^{\prime}\right)_{+} \times y^{\circ}\left(X_{r}, X_{r^{\prime}}^{\prime}\right)_{-} .
$$

Proposition 2.6 ([KuNS, Theorem 6.19]). The group $\mathrm{y}^{\circ}\left(X_{r}, X_{r^{\prime}}^{\prime}\right)_{ \pm}$is isomorphic to $\mathcal{G}_{Y}(B, y)$ by the correspondence $Y_{\mathbf{i}}(u) \mapsto y_{\mathbf{i}}(u)^{ \pm 1}, 1+$ $Y_{\mathbf{i}}(u) \mapsto 1+y_{\mathbf{i}}(u)^{ \pm 1}$ for $(\mathbf{i}, u): \mathbf{P}_{ \pm}$.

In summary, the Laurent monomials in $Y_{\mathbf{i}}(u)$ and $1+Y_{\mathbf{i}}(u)$ with $(\mathbf{i}, u): \mathbf{P}_{+}$ are embedded in the coefficient group $\mathcal{G}(B, y)$.

\subsection{Reformulation of Theorem 1.8}

We recall the periodicity theorem, originally conjectured by [RTV].

Theorem 2.7 ([Ke1, Theorem 8.2], [Ke2, Theorem 2.3]). In $\mathcal{G}(B, y)$, the following relations hold.

(i) Periodicity: $y_{\mathbf{i}}\left(u+2\left(h+h^{\prime}\right)\right)=y_{\mathbf{i}}(u)$.

(ii) Half-periodicity: $y_{i i^{\prime}}\left(u+\left(h+h^{\prime}\right)\right)=y_{\omega(i) \omega^{\prime}\left(i^{\prime}\right)}(u)$, where $\omega$ (resp., $\left.\omega^{\prime}\right)$ is the Dynkin automorphism of $X_{r}$ (resp., $\left.X_{r^{\prime}}^{\prime}\right)$ for types $A_{r}, D_{r}$ ( $r$ :odd), or $E_{6}$, and the identity otherwise. 
Proof. (i). This is due to [Ke1, Theorem 8.2] or [Ke2, Theorem 2.3]. (ii). This is obtained by combining [Ke1, Theorem 7.13] and the proof of [IIKNS, Theorem 4.27].

Let $\mathbb{R}_{+}$be the semifield of the positive real numbers by the usual multiplication and addition. By Proposition 2.6, Theorem 1.8 is equivalent to the following.

TheOREM 2.8. Let $y_{\mathbf{i}}(u) \in \mathbb{Q}_{\text {sf }}(y)(\mathbf{i} \in \mathbf{I}, u \in \mathbb{Z})$ be as above. Let $\varphi$ : $\mathbb{Q}_{\mathrm{sf}}(y) \rightarrow \mathbb{R}_{+}$be any semifield homomorphism. Then, the following identities hold.

$$
\begin{aligned}
& \frac{6}{\pi^{2}} \sum_{(\mathbf{i}, u) \in S_{+}} L\left(\frac{\varphi\left(y_{\mathbf{i}}(u)\right)}{1+\varphi\left(y_{\mathbf{i}}(u)\right)}\right)=h r r^{\prime}, \\
& \frac{6}{\pi^{2}} \sum_{(\mathbf{i}, u) \in S_{-}} L\left(\frac{\varphi\left(y_{\mathbf{i}}(u)\right)}{1+\varphi\left(y_{\mathbf{i}}(u)\right)}\right)=h^{\prime} r r^{\prime},
\end{aligned}
$$

where $S_{ \pm}=\left\{(\mathbf{i}, u) \mid \mathbf{i} \in I, 0 \leq u \leq 2\left(h+h^{\prime}\right)-1,(\mathbf{i}, u): \mathbf{P}_{ \pm}\right\}$. Also,

$$
\begin{aligned}
& \frac{6}{\pi^{2}} \sum_{(\mathbf{i}, u) \in H_{+}} L\left(\frac{\varphi\left(y_{\mathbf{i}}(u)\right)}{1+\varphi\left(y_{\mathbf{i}}(u)\right)}\right)=\frac{h r r^{\prime}}{2}, \\
& \frac{6}{\pi^{2}} \sum_{(\mathbf{i}, u) \in H_{-}} L\left(\frac{\varphi\left(y_{\mathbf{i}}(u)\right)}{1+\varphi\left(y_{\mathbf{i}}(u)\right)}\right)=\frac{h^{\prime} r r^{\prime}}{2},
\end{aligned}
$$

where $H_{ \pm}=\left\{(\mathbf{i}, u) \mid \mathbf{i} \in I, 0 \leq u \leq\left(h+h^{\prime}\right)-1,(\mathbf{i}, u): \mathbf{P}_{ \pm}\right\}$

The identities (2.12) and (2.13) are equivalent by Lemma 2.4. The identities (2.14) and (2.15) follow from (2.12) and (2.13) by the half-periodicity in Theorem 2.7 .

We are going to prove Theorem 2.8 .

\section{$\S 3$. Tropical Y-system}

Let us have an interlude to establish a property of the tropical $Y$-system (see [FZ4]) associated with the cluster algebra $\mathcal{A}(B, x, y)$ for $B=B\left(X_{r}, X_{r^{\prime}}^{\prime}\right)$.

Let $B=\left(B_{i j}\right)_{i, j \in I}$ be a general skew-symmetrizable matrix. Let $y$ be the initial coefficient tuple of the cluster algebra $\mathcal{A}(B, x, y)$ with coefficients in the universal semifield $\mathbb{Q}_{\mathrm{sf}}(y)$. The tropical semifield $\operatorname{Trop}(y)$ is an abelian 
multiplicative group freely generated by the elements $y_{i}(i \in I)$ with the addition $\oplus$ :

$$
\prod_{i \in I} y_{i}^{a_{i}} \oplus \prod_{i \in I} y_{i}^{b_{i}}=\prod_{i \in I} y_{i}^{\min \left(a_{i}, b_{i}\right)} .
$$

The image of $f \in \mathbb{Q}_{\text {sf }}(y)$ by the natural projection $\mathbb{Q}_{s f}(y) \rightarrow \operatorname{Trop}(y)$ is denoted by $[f]_{\mathrm{T}}$ and called the tropical evaluation of $f$ (see [FZ4]).

We say a (Laurent) monomial in $y=\left(y_{i}\right)_{1 \in I}$ is positive if its exponents are all nonnegative and at least one of them is positive. A negative monomial is defined similarly.

LEMmA 3.1. Suppose that $y^{\prime \prime}$ is the coefficient tuple obtained from the mutation of another coefficient tuple $y^{\prime}$ at $k$. Then, for any $i \neq k,\left[y_{i}^{\prime \prime}\right]_{\mathrm{T}}=$ $\left[y_{i}^{\prime}\right]_{\mathrm{T}}$ if one of the following conditions holds.

(i) $B_{k i}=0$.

(ii) $B_{k i}>0$, and $\left[y_{k}^{\prime}\right]_{\mathrm{T}}$ is negative.

(iii) $B_{k i}<0$, and $\left[y_{k}^{\prime}\right]_{\mathrm{T}}$ is positive.

Proof. This is an immediate consequence of the exchange relation (2.2).

Now we claim a key proposition in our proof of Theorem 2.8.

Proposition 3.2. For the cluster algebra $\mathcal{A}(B, x, y)$ for $B=B\left(X_{r}, X_{r^{\prime}}^{\prime}\right)$, the following properties hold.

(i) The tropical evaluation $\left[y_{\mathbf{i}}(u)\right]_{\mathrm{T}}$ of $y_{\mathbf{i}}(u)(\mathbf{i} \in \mathbf{I}, u \in \mathbb{Z})$ is a positive or negative monomial in $y=y(0)$.

(ii) For $0 \leq u \leq h^{\prime}-1$ and $(\mathbf{i}, u): \mathbf{P}_{+},\left[y_{\mathbf{i}}(u)\right]_{\mathrm{T}}$ is a positive monomial. For $-h \leq u \leq-1$ and $(\mathbf{i}, u): \mathbf{P}_{+},\left[y_{\mathbf{i}}(u)\right]_{\mathrm{T}}$ is a negative monomial.

(iii) Let $N_{+}$(resp., $N_{-}$) be the number of the positive (resp., negative) monomials $\left[y_{\mathbf{i}}(u)\right]_{\mathrm{T}}\left((\mathbf{i}, u) \in S_{+}\right)$, where $S_{+}$is the domain in Theorem 2.8. Then,

$$
N_{+}=h^{\prime} r r^{\prime}, \quad N_{-}=h r r^{\prime}
$$

The properties (i) and (iii) follow from (ii) by the half-periodicity in Theorem 2.7. In the case $X_{r^{\prime}}^{\prime}=A_{1}$ or $X_{r}=A_{1}$, that is, the "level 2 case" or its level-rank dual in the original context, Proposition 3.2 reduces to the known one for the cluster algebra of finite type (see [FZ4, Proposition 10.7]).

Before giving a proof, it is instructive to observe some examples. 
ExAmPLE 3.3. Let $X_{r}=A_{1}, I_{+}=\{1\}, I_{-}=\emptyset$, and let $X_{r^{\prime}}^{\prime}=A_{2}, I_{+}^{\prime}=$ $\{1\}, I_{-}^{\prime}=\{2\}$. We have $h=2$ and $h^{\prime}=3$. We visualize the mutation matrix $B$ by a quiver in the correspondence

$$
\mathbf{i} \rightarrow \mathbf{j} \quad \Longleftrightarrow \quad B_{\mathrm{ij}}=1
$$

Set $y_{1}:=y_{11}$, and set $y_{2}:=y_{12}$. Then, $\left[y_{\mathbf{i}}(u)\right]_{\mathrm{T}}$ for $0 \leq u \leq 5$ is given as follows:

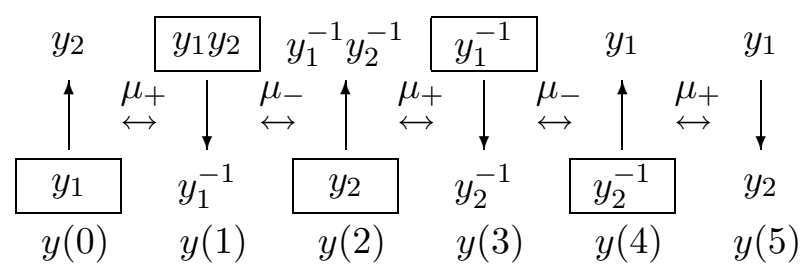

Here, the framed variables are all the elements in the domain $H_{+}$. Certainly, we have $N_{+} / 2=3$ and $N_{-} / 2=2$, which agree with (3.2). Moreover, we observe that the positive monomials occur consecutively for $0 \leq u \leq 2$. This is a consequence of [FZ4, Proposition 10.7]. In fact, they correspond to the positive roots $\alpha_{1}, \alpha_{1}+\alpha_{2}, \alpha_{2}$ of $A_{2}$. We abbreviate the above diagram as follows:

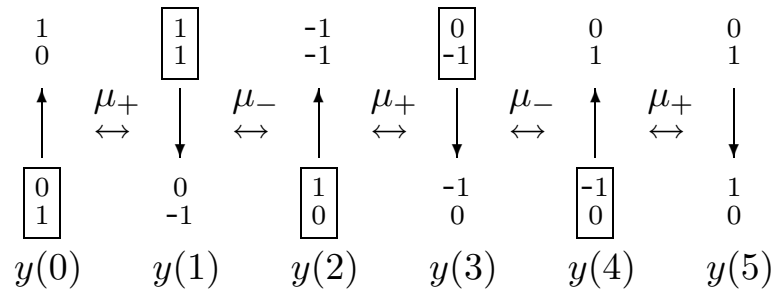

EXAmple 3.4. Let $X_{r}=A_{3}, I_{+}=\{1,3\}, I_{-}=\{2\}$, and let $X_{r^{\prime}}^{\prime}=A_{1}$, $I_{+}^{\prime}=\{1\}, I_{-}^{\prime}=\emptyset$. We have $h=4$ and $h^{\prime}=2$. We consider mutations for $-6 \leq u \leq 0$ by moving in the reverse direction of $u$. The result is abbreviated in the following diagram:

$$
\begin{aligned}
& \begin{aligned}
001 \leftarrow & 010 \rightarrow 100 \stackrel{\mu_{+}}{\leftrightarrow} \quad 00-1 \rightarrow \frac{010}{y(-6)} \\
y(-5) & -100
\end{aligned}
\end{aligned}
$$

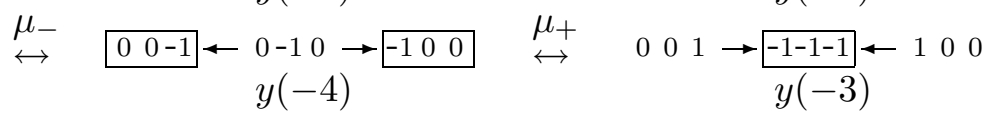

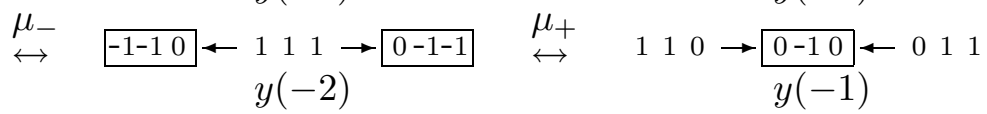

$$
\begin{aligned}
& \stackrel{\mu_{-}}{\leftrightarrow} 100 \leftarrow 010 \rightarrow 001 \\
& y(0)
\end{aligned}
$$


Here, 110, for example, represents the monomial $y_{11} y_{21}$. The framed variables correspond to all the elements in the domain $H_{+}$modulo half-period $h+h^{\prime}=6$. Certainly, we have $N_{+} / 2=3$ and $N_{-} / 2=6$, which agree with (3.2). We observe that the negative monomials occur consecutively for $-4 \leq$ $u \leq-1$. Again, this is a consequence of [FZ4, Proposition 10.7], and they correspond to the positive roots of $A_{3}$.

Now we are ready to proceed to a "higher" level. The next example looks like a toy example, but it completely clarifies why Proposition 3.2 holds.

EXAmple 3.5. Let $X_{r}=A_{3}, I_{+}=\{1,3\}, I_{-}=\{2\}$, and let $X_{r^{\prime}}^{\prime}=A_{2}$, $I_{+}^{\prime}=\{1\}, I_{-}^{\prime}=\{2\}$. We have $h=4$ and $h^{\prime}=3$. We consider mutations for $-4 \leq u \leq 3$ by moving in both directions of $u$. The result is shown in Figure 1. The framed variables correspond to all the elements in the domain $H_{+}$modulo half-period $h+h^{\prime}=7$, and

$$
\begin{array}{lll}
0 & 0 & 0 \\
1 & 1 & 0
\end{array}
$$

for example, represents the monomial $y_{11} y_{12}$. Certainly, we have $N_{+} / 2=$ 9 and $N_{-} / 2=12$, which agree with (3.2). We observe that the positive monomials occur consecutively for $0 \leq u \leq 2$, while the negative monomials do so for $-4 \leq u \leq-1$.

When we look at Figure 1 more closely, we find a remarkable factorization property of the tropical Y-system, which does not occur in the nontropical Y-system. First, examine the region $0 \leq u \leq 2$. Then, the contents in the left and right columns for $y(u)$ mutate exactly in the same pattern as in Example 3.3. So does the middle column with the other choice of bipartite decomposition of $I^{\prime}$. In particular, there is no interaction in the horizontal direction. This is because property (iii) in Lemma 3.1 is satisfied at any mutation point $\mathbf{k}$ and any $\mathbf{i}$ horizontally adjacent to $\mathbf{k}$. On the other hand, in the region $-4 \leq u \leq-1$, the contents in the lower row mutate exactly in the same pattern as in Example 3.4. So does the upper row with the other choice of bipartite decomposition of $I$. Now, there is no interaction in the vertical direction. Again, this is because property (iii) in Lemma 3.1 is satisfied at any mutation point $\mathbf{k}$ and any $\mathbf{i}$ vertically adjacent to $\mathbf{k}$. (Note that $(\mathbf{k}, u): \mathbf{P}_{-}$for mutations in the reverse direction of $u$.)

Proof of Proposition 3.2. It is enough to prove (ii). We just repeat the argument in Example 3.5 in a general manner. Let us recall the definition 


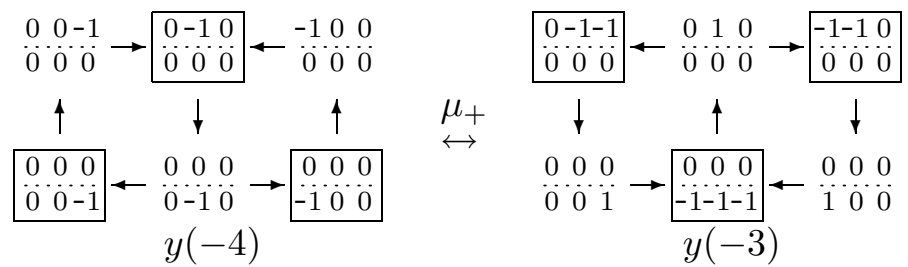

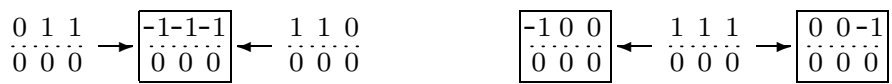

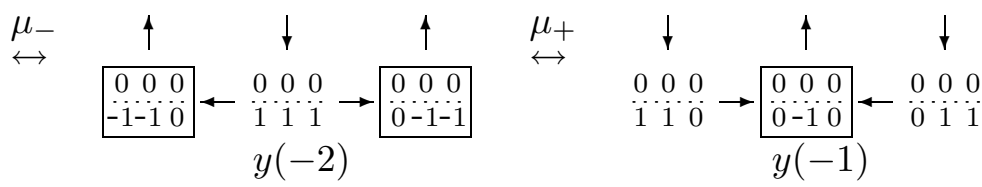

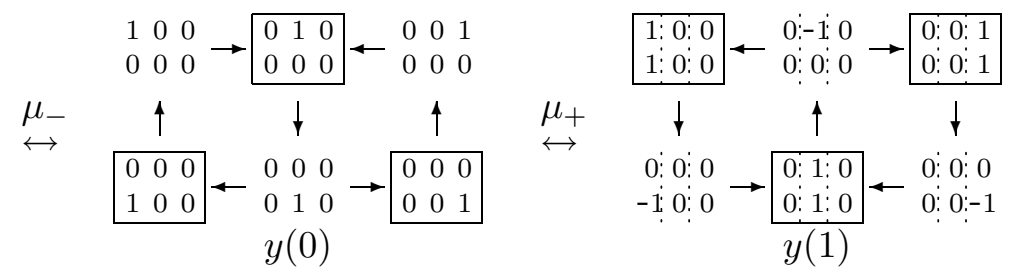

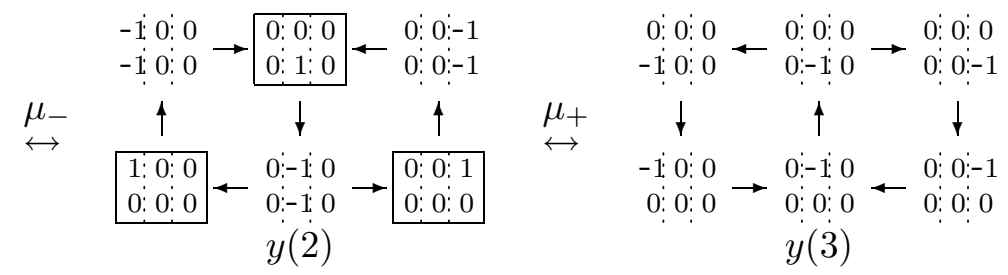

Figure 1: Tropical Y-system for $X_{r}=A_{3}$ and $X_{r^{\prime}}^{\prime}=A_{2}$

of the integer vector $\mathbf{d}(i, u)$ in [FZ4, Definition 10.2] (in our notation). Let $s_{1}, \ldots, s_{r}$ be the simple reflections of the Weyl group of type $X_{r}$, and let

$$
t_{+}=\prod_{i \in I_{+}} s_{i}, \quad t_{-}=\prod_{i \in I_{-}} s_{i} .
$$

Define the piecewise-linear analogue $\tau_{ \pm}$of $t_{ \pm}$acting on the set $\Phi_{\geq-1}$ of all the positive roots and the negative simple roots of $X_{r}$ by

$$
\tau_{ \pm}(\alpha)= \begin{cases}-\alpha_{i} & \alpha=-\alpha_{i}, i \in I_{\mp} \\ t_{ \pm}(\alpha) & \text { otherwise. }\end{cases}
$$


Then, $\mathbf{d}(i, u)$ is defined by

$$
\mathbf{d}(i, u)= \begin{cases}\left(\tau_{-} \tau_{+}\right)^{u / 2}\left(-\alpha_{i}\right) & i \in I_{+} \text {and even } u \geq 0 \\ \left(\tau_{-} \tau_{+}\right)^{(u-1) / 2} \tau_{-}\left(-\alpha_{i}\right) & i \in I_{-} \text {and odd } u \geq 0\end{cases}
$$

It is known by [FZ4, Proposition 9.3] that $\mathbf{d}(i, u)$ is a positive root of $X_{r}$ for $1 \leq u \leq h$. We naturally identify $\mathbf{d}(i, u)=\sum_{k \in I} d(i, u)_{k} \alpha_{k}$ with the integer vector $\left(d(i, u)_{k}\right)_{k \in I}$.

(1) The case $0 \leq u \leq h^{\prime}-1$. Let $\mathbf{d}\left(i^{\prime}, u\right)=\left(d\left(i^{\prime}, u\right)_{k^{\prime}}\right)_{k^{\prime} \in I^{\prime}}$ be the integer vector as above for $X_{r^{\prime}}^{\prime}$ with $I^{\prime}=I_{+}^{\prime} \sqcup I_{-}^{\prime}$. Let $\tilde{\mathbf{d}}\left(i^{\prime}, u\right)$ be the same vector but for the opposite choice of the bipartite decomposition of $I^{\prime}$. Forget temporarily all the arrows in the horizontal direction. Then, one has

$$
\begin{aligned}
& {\left[y_{i i^{\prime}}(u)\right]_{\mathrm{T}}=\prod_{k^{\prime} \in I^{\prime}} y_{i k^{\prime}}^{d\left(\omega^{\prime}\left(i^{\prime}\right), h^{\prime}-u\right)_{k^{\prime}},}, \quad(\mathbf{i}, u): \mathbf{P}_{+}, i \in I_{+},} \\
& {\left[y_{i i^{\prime}}(u)\right]_{\mathrm{T}}=\prod_{k^{\prime} \in I^{\prime}} y_{i k^{\prime}} \tilde{d}\left(\omega^{\prime}\left(i^{\prime}\right), h^{\prime}-u\right)_{k^{\prime}}, \quad(\mathbf{i}, u): \mathbf{P}_{+}, i \in I_{-},}
\end{aligned}
$$

by applying [FZ4, Proposition 10.7] in our convention. This remains true even in the presence of the horizontal arrows (factorization property), because of Lemma 3.1 and the positivity of vectors $\mathbf{d}\left(i^{\prime}, u\right)$ and $\tilde{\mathbf{d}}\left(i^{\prime}, u\right)$.

(2) The case $-h \leq u \leq-1$. We consider mutations in the reverse direction of $u$ at points $(\mathbf{i}, u): \mathbf{P}_{-}$. Let $\mathbf{d}(i, u)$ be the corresponding vector for $X_{r}$ with $I=I_{+} \sqcup I_{-}$, and let $\tilde{\mathbf{d}}(i, u)$ be the one for the opposite choice of the bipartite decomposition of $I$. Then, repeating the same argument, and also using Lemma 2.4(1), we obtain

$$
\begin{aligned}
& {\left[y_{i i^{\prime}}(u)\right]_{\mathrm{T}}=\prod_{k \in I} y_{k i^{\prime}}^{-d(\omega(i), h+u+1)_{k}}, \quad(\mathbf{i}, u): \mathbf{P}_{+}, i^{\prime} \in I_{-}^{\prime},} \\
& {\left[y_{i i^{\prime}}(u)\right]_{\mathrm{T}}=\prod_{k \in I} y_{k i^{\prime}}^{d(\omega(i), h+u)_{k}}, \quad(\mathbf{i}, u): \mathbf{P}_{-}, i^{\prime} \in I_{-}^{\prime},} \\
& {\left[y_{i i^{\prime}}(u)\right]_{\mathrm{T}}=\prod_{k \in I} y_{k i^{\prime}}^{-\tilde{d}(\omega(i), h+u+1)_{k}}, \quad(\mathbf{i}, u): \mathbf{P}_{+}, i^{\prime} \in I_{+}^{\prime},} \\
& {\left[y_{i i^{\prime}}(u)\right]_{\mathrm{T}}=\prod_{k \in I} y_{k i^{\prime}}^{\tilde{d}(\omega(i), h+u)_{k}}, \quad(\mathbf{i}, u): \mathbf{P}_{-}, i^{\prime} \in I_{+}^{\prime}}
\end{aligned}
$$

This completes the proof of Proposition 3.2. 


\section{§4. Proof of Theorem 2.8}

We prove Theorem 2.8 by applying the method of [FS] with a mixture of ideas by $[\mathrm{CGT}]$ and $[\mathrm{C}]$.

The proof is divided into two steps. First, we prove that the left-hand side of (2.12) is independent of a semifield homomorphism $\varphi$ (constancy or rigidity property). Next, we evaluate its value at the $0 / \infty$ limit.

\subsection{Constancy property}

Let $A$ be a multiplicative abelian group. The group $A \otimes_{\mathbb{Z}} A$ is the additive abelian group generated by $g \otimes h(g, h \in A)$ with relations

$$
(f g) \otimes h=f \otimes h+g \otimes h, \quad h \otimes(f g)=h \otimes f+h \otimes g .
$$

As a consequence, we also have the following relations:

$$
\begin{gathered}
1 \otimes h=h \otimes 1=0, \\
f^{-1} \otimes g=-f \otimes g, \quad g \otimes f^{-1}=-g \otimes f .
\end{gathered}
$$

Let $S^{2} A$ be the subgroup of $A \otimes_{\mathbb{Z}} A$ generated by $f \otimes f(f \in A)$, and let $\bigwedge^{2} A$ be the quotient of $A \otimes_{\mathbb{Z}} A$ by $S^{2} A$. In $\bigwedge^{2} A$ we use $\wedge$ instead of $\otimes$.

According to a very general theorem by [FS, Proposition 1] (see also [B], [Zag1]), the constancy property of the left-hand side of (2.12) follows from the following fact.

Proposition 4.1. In $\bigwedge^{2} \mathbb{Q}_{\mathrm{sf}}(y)$, we have

$$
\sum_{(\mathbf{i}, u) \in S_{+}} y_{\mathbf{i}}(u) \wedge\left(1+y_{\mathbf{i}}(u)\right)=0 .
$$

Motivated by [CGT] and [C], we use the F-polynomials of [FZ4] to prove Proposition 4.1.

The $F$-polynomial $F_{\mathbf{i}}(u) \in \mathbb{Q}_{\mathrm{sf}}(y)$ at $(\mathbf{i}, u)(\mathbf{i} \in \mathbf{I}, u \in \mathbb{Z})$ is defined by the specialization of $\left[x_{\mathbf{i}}(u)\right]_{\mathrm{T}}$ at $x_{\mathbf{j}}=x_{\mathbf{j}}(0)=1(\mathbf{j} \in \mathbf{I})$. (Caution: do not make the tropical evaluation for the addition in $\mathbb{Q P}(x)$ !) It is represented as a polynomial in $y$ with integer coefficients due to the Laurent phenomenon (see [FZ4, Proposition 3.6]). 
For our matrix $B=B\left(X_{r}, X_{r^{\prime}}^{\prime}\right)$, it is convenient to define the incidence matrices $M=\left(M_{\mathbf{i j}}\right)_{\mathbf{i}, \mathbf{j} \in \mathbf{I}}$ and $M^{\prime}=\left(M_{\mathbf{i j}}^{\prime}\right)_{\mathbf{i}, \mathbf{j} \in \mathbf{I}}$ as

$$
M_{\mathbf{i j}}=\left\{\begin{array}{ll}
1 & i \sim j, i^{\prime}=j^{\prime}, \\
0 & \text { otherwise }
\end{array} \quad M_{\mathbf{i j}}^{\prime}= \begin{cases}1 & i=j, i^{\prime} \sim j^{\prime} \\
0 & \text { otherwise }\end{cases}\right.
$$

Note that they are symmetric matrices.

Lemma 4.2. (i) For $(\mathbf{i}, u): \mathbf{P}_{+}$, the following relations hold in $\mathbb{Q}_{s f}(y)$ :

$$
\begin{aligned}
F_{\mathbf{i}}(u)= & F_{\mathbf{i}}(u-1), \\
F_{\mathbf{i}}(u-1) F_{\mathbf{i}}(u+1)= & {\left[\frac{y_{\mathbf{i}}(u)}{1+y_{\mathbf{i}}(u)}\right]_{\mathrm{T}} \prod_{\mathbf{j} \in \mathbf{I}} F_{\mathbf{j}}(u)^{M_{\mathbf{j i}}} } \\
& +\left[\frac{1}{1+y_{\mathbf{i}}(u)}\right]_{\mathrm{T}} \prod_{\mathbf{j} \in \mathbf{I}} F_{\mathbf{j}}(u)^{M_{\mathbf{j i}}^{\prime}} \\
y_{\mathbf{i}}(u)= & {\left[y_{\mathbf{i}}(u)\right]_{\mathrm{T}} \frac{\prod_{\mathbf{j} \in \mathbf{I}} F_{\mathbf{j}}(u)^{M_{\mathbf{j i}}}}{\prod_{\mathbf{j} \in \mathbf{I}} F_{\mathbf{j}}(u)^{M_{\mathbf{j i}}^{\prime}}} }
\end{aligned}
$$

(ii) Periodicity: $F_{\mathbf{i}}\left(u+2\left(h+h^{\prime}\right)\right)=F_{\mathbf{i}}(u)$.

(iii) Each polynomial $F_{\mathbf{i}}(u)$ has constant term 1.

Proof. (i). The first two relations follow from (2.3). The last one is due to [FZ4, Proposition 3.13]. (ii). This was shown by [Ke1] and [Ke2]. (iii). For $u=0$, this is true by $F_{\mathbf{i}}(0)=1$. Then, the claim is shown by induction on $u$, by using (4.6), (4.7), and Proposition 3.2(i) (see [FZ4, Proposition 5.6]).

Remark 4.3. Lemma 4.2(iii) is also true by [DWZ, Theorem 1.7]. Then, Proposition 3.2(i) is a consequence of Lemma 4.2(iii) due to [FZ4, Proposition 5.6].

By (4.7) and (4.8), we also have, for $(\mathbf{i}, u): \mathbf{P}_{+}$,

$$
1+y_{\mathbf{i}}(u)=\left[1+y_{\mathbf{i}}(u)\right]_{\mathrm{T}} \frac{F_{\mathbf{i}}(u-1) F_{\mathbf{i}}(u+1)}{\prod_{\mathbf{j} \in \mathbf{I}} F_{\mathbf{j}}(u)^{M_{\mathbf{j i}}^{\prime}}} .
$$

Now, we put (4.8) and (4.9) into (4.4) and expand it.

First,

$$
\sum_{(\mathbf{i}, u) \in S_{+}}\left[y_{\mathbf{i}}(u)\right]_{\mathrm{T}} \wedge\left[1+y_{\mathbf{i}}(u)\right]_{\mathrm{T}}=0
$$


since each monomial $\left[y_{\mathbf{i}}(u)\right]_{\mathrm{T}}$ is either positive or negative by Proposition 3.2 .

Second, the contributions from the terms involving only $F_{\mathbf{i}}(u)$ vanish due to the symmetry argument of [CGT, Section 3]. For example,

$$
\begin{aligned}
& \sum_{(\mathbf{i}, u) \in S_{+}} \prod_{\mathbf{j} \in \mathbf{I}} F_{\mathbf{j}}(u)^{M_{\mathbf{j i}}} \wedge F_{\mathbf{i}}(u-1) F_{\mathbf{i}}(u+1) \\
& =\sum_{(\mathbf{i}, u) \in S_{+}} F_{\mathbf{i}}(u-1) F_{\mathbf{i}}(u+1) \wedge \prod_{\mathbf{j} \in \mathbf{I}} F_{\mathbf{j}}(u)^{M_{\mathbf{j i}}}
\end{aligned}
$$

by changing the variables twice; therefore, it vanishes.

Third, the contributions from the remaining five terms cancel due to the Y-system (1.10):

$$
\begin{gathered}
\sum_{(\mathbf{i}, u) \in S_{+}}\left[y_{\mathbf{i}}(u)\right]_{\mathrm{T}} \wedge F_{\mathbf{i}}(u-1)=\sum_{(\mathbf{i}, u) \in S_{-}}\left[y_{\mathbf{i}}(u+1)\right]_{\mathrm{T}} \wedge F_{\mathbf{i}}(u), \\
\sum_{(\mathbf{i}, u) \in S_{+}}\left[y_{\mathbf{i}}(u)\right]_{\mathrm{T}} \wedge F_{\mathbf{i}}(u+1)=\sum_{(\mathbf{i}, u) \in S_{-}}\left[y_{\mathbf{i}}(u-1)\right]_{\mathrm{T}} \wedge F_{\mathbf{i}}(u), \\
-\sum_{(\mathbf{i}, u) \in S_{+}}\left[y_{\mathbf{i}}(u)\right]_{\mathrm{T}} \wedge \prod_{\mathbf{j} \in \mathbf{I}} F_{\mathbf{j}}(u)^{M_{\mathbf{j} \mathbf{i}}^{\prime}}=\sum_{(\mathbf{i}, u) \in S_{-}} \prod_{\mathbf{j} \in \mathbf{I}}\left[y_{\mathbf{j}}(u)\right]_{\mathrm{T}}^{-M_{\mathbf{j i}}^{\prime}} \wedge F_{\mathbf{i}}(u), \\
-\sum_{(\mathbf{i}, u) \in S_{+}}\left[1+y_{\mathbf{i}}(u)\right]_{\mathrm{T}} \wedge \prod_{\mathbf{j} \in \mathbf{I}} F_{\mathbf{j}}(u)^{M_{\mathbf{j} \mathbf{i}}} \\
=\sum_{(\mathbf{i}, u) \in S_{-}} \prod_{\mathbf{j} \in \mathbf{I}}\left[1+y_{\mathbf{j}}(u)\right]_{\mathrm{T}}^{-M_{\mathbf{j i}}} \wedge F_{\mathbf{i}}(u), \\
-\sum_{(\mathbf{i}, u) \in S_{+}}\left[1+y_{\mathbf{i}}(u)\right]_{\mathrm{T}} \wedge \prod_{\mathbf{j} \in \mathbf{I}} F_{\mathbf{j}}(u)^{-M_{\mathbf{j} \mathbf{i}}^{\prime}} \\
=\sum_{(\mathbf{i}, u) \in S_{-}} \prod_{\mathbf{j} \in \mathbf{I}}\left[1+y_{\mathbf{j}}(u)\right]_{\mathrm{T}}^{M_{\mathbf{j i}}^{\prime}} \wedge F_{\mathbf{i}}(u) .
\end{gathered}
$$

This completes the proof of Proposition 4.1.

REMARK 4.4. Equation (4.4) was studied in [CGT, (3.32)] by using the parameterization of $y_{\mathbf{i}}(u)$ by the T-system. However, it is known that this does not give a "general" solution of the Y-system (see [IIKNS, remark after Proposition 3.8]). Therefore, we use F-polynomials instead of the T-system. 


\subsection{Evaluation in the $0 / \infty$ limit}

Following [C], we evaluate the value of the left-hand side of $(2.12)$ in the limit such that each $\varphi\left(y_{\mathbf{i}}(u)\right)$ goes to either zero or infinity (the $0 / \infty$ limit). Then, the value is equal to the number of the variables which go to infinity due to (1.2).

Thanks to Proposition 3.2, we already have such a limit at hand. Take the one-parameter family of semifield homomorphisms $\varphi_{t}: \mathbb{Q}_{s f}(y) \rightarrow \mathbb{R}_{+}(0<$ $t<1)$ defined by $\varphi_{t}\left(y_{\mathbf{i}}\right)=t$ for any $\mathbf{i} \in I$. Then, in the limit $\rightarrow 0, \varphi_{t}\left(y_{\mathbf{i}}(u)\right)$ is zero if $\left[y_{\mathbf{i}}(u)\right]_{\mathrm{T}}$ is positive and $\infty$ if $\left[y_{\mathbf{i}}(u)\right]_{\mathrm{T}}$ is negative, due to (4.8) and Lemma 4.2(iii). Therefore, the value of the left-hand side of (2.12) is $N_{-}=h r r^{\prime}$ by Proposition 3.2.

This completes the proof of Theorem 2.8.

Acknowledgment. It is my great pleasure to thank Atsuo Kuniba for sharing his insight into the dilogarithm identities for many years, and also for useful comments on the manuscript.

\section{REFERENCES}

[BR1] V. V. Bazhanov and N. Y. Reshetikhin, Critical RSOS models and conformal field theory, Internat. J. Modern Phys. A 4 (1989), 115-142.

[BR2] - Restricted solid-on-solid models connected with simply laced algebras and conformal field theory, J. Phys. A 23 (1990), 1477-1492.

[B] S. Bloch, "Applications of the dilogarithm function in algebraic K-theory and algebraic geometry" in Proceedings of the International Symposium on Algebraic Geometry (Kyoto, 1977), Kinokuniya, Tokyo, 1978, 103-114.

[CGT] R. Caracciolo, F. Gliozzi, and R. Tateo, A topological invariant of $R G$ flows in $2 D$ integrable quantum field theories, Internat. J. Modern Phys. 13 (1999), 2927-2932.

[C] F. Chapoton, Functional identities for the Rogers dilogarithm associated to cluster Y-systems, Bull. Lond. Math. Soc. 37 (2005), 755-760.

[DWZ] H. Derksen, J. Weyman, and A. Zelevinsky, Quivers with potentials and their representations II: Applications to cluster algebras, J. Amer. Math. Soc. 23 (2010), 749-790.

[DS] J. L. Dupont and C.-H. Sah, Dilogarithm identities in conformal field theory and group homology, Comm. Math. Phys. 161 (1994), 265-282.

[FZ] V. A. Fateev and A. B. Zamolodchikov, Nonlocal (parafermion) currents in twodimensional conformal quantum field theory and self-dual critical points in $Z_{N^{-}}$ symmetric statistical systems, J. Exp. Theor. Phys. 62 (1985), 215-225.

[FG] V. V. Fock and A. B. Goncharov, Cluster ensembles, quantization and the dilogarithm, Ann. Sci. Éc. Norm. Supér. (4) 39 (2009), 865-930.

[FZ1] S. Fomin and A. Zelevinsky, Cluster algebras, I: Foundations, J. Amer. Math. Soc. 15 (2002), 497-529.

[FZ2] — Cluster algebras, II: Finite type classification, Invent. Math. 154 (2003), 63-121. 
[FZ3] - Y-systems and generalized associahedra, Ann. of Math. (2) 158 (2003), 977-1018.

[FZ4] - Cluster algebras, IV: Coefficients, Compos. Math. 143 (2007), 112-164.

[FS] E. Frenkel and A. Szenes, Thermodynamic Bethe ansatz and dilogarithm identities, I, Math. Res. Lett. 2 (1995), 677-693.

[G] D. Gepner, New conformal field theories associated with Lie algebras and their partition functions, Nuclear Phys. B 290 (1987), 10-24.

[GW] D. Gepner and E. Witten, String theory on group manifolds, Nuclear Phys. B 278 (1986), 493-549.

[GT1] F. Gliozzi and R. Tateo, ADE functional dilogarithm identities and integrable models, Phys. Lett. B 348 (1995), 677-693.

[GT2] - Thermodynamic Bethe ansatz and three-fold triangulations, Internat. J. Modern Phys. A 11 (1996), 4051-4064.

[HW] H. C. Hutchins and H. J. Weinert, Homomorphisms and kernels of semifields, Period. Math. Hungar. 21 (1990), 113-152.

[IIKKN1] R. Inoue, O. Iyama, B. Keller, A. Kuniba, and T. Nakanishi, Periodicities of $\mathrm{T}$ and $\mathrm{Y}$-systems, dilogarithm identities, and cluster algebras, I: Type $B_{r}$, preprint, arXiv:1001.1880 [math.QA]

[IIKKN2] - Periodicities of $\mathrm{T}$ and $\mathrm{Y}$-systems, dilogarithm identities, and cluster algebras, II: Types $C_{r}, F_{4}$, and $G_{2}$, preprint, arXiv:1001.1881 [math.QA]

[IIKNS] R. Inoue, O. Iyama, A. Kuniba, T. Nakanishi, and J. Suzuki, Periodicities of T-systems and Y-systems, Nagoya Math. J. 197 (2010), 59-174.

[Ke1] B. Keller, Cluster algebras, quiver representations and triangulated categories, preprint, arXiv:0807.1960 [math.RT]

[Ke2] - The periodicity conjecture for pairs of Dynkin diagrams, preprint, arXiv:1001.1531 [math.RT]

[K1] A. N. Kirillov, Identities for the Rogers dilogarithm function connected with simple Lie algebras, J. Soviet Math. 47 (1989), 2450-2458.

[K2] - Dilogarithm identities, Progr. Theoret. Phys. Suppl. 118 (1995), 61-142.

[KR1] A. N. Kirillov and N. Y. Reshetikhin, Exact solution of the Heisenberg XXZ model of spin s, J. Soviet Math. 35 (1986), 2627-2643.

[KR2] - Representations of Yangians and multiplicities of the inclusion of the irreducible components of the tensor product of representations of simple Lie algebras, J. Soviet Math. 52 (1990), 3156-3164.

[KnZ] V. G. Knizhnik and A. B. Zamolodchikov, Current algebra and Wess-Zumino model in two dimensions, Nuclear Phys. B 247 (1984), 83-103.

[Ku] A. Kuniba, Thermodynamics of the $U_{q}\left(X_{r}^{(1)}\right)$ Bethe ansatz system with $q$ a root of unity, Nuclear Phys. B 389 (1993), 209-244.

$[\mathrm{KuN}]$ A. Kuniba and T. Nakanishi, Spectra in conformal field theories from the Rogers dilogarithm, Modern Phys. Lett. A 7 (1992), 3487-3494.

[KuNS] A. Kuniba, T. Nakanishi, and J. Suzuki, T-systems and Y-systems for quantum affinizations of quantum Kac-Moody algebras, SIGMA Symmetry Integrability Geom. Methods Appl. 5 (2009), 1-23.

[L] L. Lewin, Polylogarithms and Associated Functions, North-Holland, Amsterdam, 1981.

[N] W. Nahm, "Conformal field theory and torsion elements of the Bloch group" in Frontiers in Number Theory, Physics, and Geometry, II, Springer, Berlin, 2007, 67132 . 
[NK] W. Nahm and S. Keegan, Integrable deformations of CFTs and the discrete Hirota equations, preprint, arXiv.0905.3776 [hep-th]

[NRT] W. Nahm, A. Recknagel, and M. Terhoeven, Dilogarithm identities in conformal field theory, Modern Phys. Lett. A 8 (1993), 1835-1847.

[RTV] F. Ravanini, R. Tateo, and A. Valleriani, Dynkin TBA's, Internat. J. Modern Phys. A 8 (1993), 1707-1727.

[RS] B. Richmond and G. Szekeres, Some formulas related to dilogarithm, the zeta function and the Andrews-Gordon identities, J. Aust. Math. Soc. 31 (1981), 362-373.

[S] A. Szenes, Periodicity of Y-systems and flat connections, Lett. Math. Phys. 89 (2009), 217-230.

[V] A. Y. Volkov, On the periodicity conjecture for Y-systems, Comm. Math. Phys. 276 (2007), 509-517.

[Zag1] D. Zagier, "Polylogarithms, Dedekind zeta functions, and the algebraic K-theory of fields" in Arithmetic Algebraic Geometry, Progr. Math. 89, Birkhäuser, Boston, 1990, 391-430.

[Zag2] - "The dilogarithm function" in Frontiers in Number Theory, Physics, and Geometry, II, Springer, Berlin, 2007, 3-65.

[Zam] A. B. Zamolodchikov, On the thermodynamic Bethe ansatz equations for reflectionless ADE scattering theories, Phys. Lett. B 253 (1991), 391-394.

Graduate School of Mathematics

Nagoya University

Nagoya 464-8604

Japan

nakanishi@math.nagoya-u.ac.jp 